Cahiers de philosophie de l'université de

\title{
Le conventionnalisme géométrique, une alternative radicale au réalisme spatial
}

Le contexte français du conventionnalisme géométrique de Poincaré

Philippe Nabonnand

\section{OpenEdition}

\section{Journals}

Édition électronique

URL : https://journals.openedition.org/cpuc/1293

DOI : $10.4000 /$ cpuc. 1293

ISSN : 2677-6529

\section{Éditeur}

Presses universitaires de Caen

Édition imprimée

Date de publication : 15 décembre 2008

Pagination : 63-86

ISBN : 978-2-84133-332-5

ISSN : $1282-6545$

\section{Référence électronique}

Philippe Nabonnand, «Le conventionnalisme géométrique, une alternative radicale au réalisme spatial », Cahiers de philosophie de l'université de Caen [En ligne], 45 | 2008, mis en ligne le 04 septembre 2020, consulté le 31 janvier 2023. URL : http://journals.openedition.org/cpuc/1293 ; DOI : https:// doi.org/10.4000/cpuc. 1293

Creative Commons - Attribution - Pas d'Utilisation Commerciale 4.0 International - CC BY-NC 4.0 https://creativecommons.org/licenses/by-nc/4.0/ 


\section{LE CONVENTIONNALISME GÉOMETRIQUE, UNE ALTERNATIVE RADICALE AU RÉALISME SPATIAL. Le contexte français du conventionnalisme géométrique de Poincaré}

Le conventionnalisme géométrique de Poincaré est bien connu ${ }^{1}$; les axiomes de la géométrie sont des conventions, le problème de la vérité de la géométrie n'a pas de sens et on doit le remplacer par celui de la commodité de la géométrie. Le conventionnalisme géométrique de Poincaré est associé à une théorie psycho-physio-logicomathématique de la genèse de la géométrie et de l'espace:

Je me suis demandé quel est le véritable caractère des vérités géométriques et en particulier du postulatum d'Euclide. [...] J'ai recherché également à analyser l'origine psychologique de la notion d'espace ${ }^{2}$.

Poincaré cite le mathématicien norvégien Lie comme référence essentielle pour la théorie des groupes de transformations et le physicien-physiologiste allemand Helmholtz comme source d'information concernant la psycho-physiologie. Comme l'attestent ses propres travaux mathématiques et sa correspondance, il est indéniable que Poincaré a étudié les travaux de Lie; il a certainement aussi lu certains travaux de psycho-physiologie de Helmholtz; la traduction en français de l'Optique physiologique a été publiée en 1867. On ne peut pourtant simplement se contenter de considérer la genèse des travaux de philosophie de l'espace de Poincaré dans cette seule perspective. En effet, dans son article Sur les hypothèses

1. Pour se rendre compte de l'actualité du conventionnalisme de Poincaré, il n'est besoin que de consulter la bibliographie des travaux sur Poincaré réalisée par les Archives Poincaré. On pourra se reporter aussi à Nabonnand 2000a et Nabonnand 2005.

2. Poincaré 1901. 
fondamentales de la géométrie publié en 1887, dans lequel il présente pour la première fois les thèses essentielles de son conventionnalisme géométrique, Poincaré conclut après avoir affirmé que "les hypothèses fondamentales de la géométrie ne sont pas des faits expérimentaux" mais que "c'est cependant l'observation de certains phénomènes physiques qui les fait choisir parmi toutes les hypothèses possibles":

Je n'insiste pas davantage, car le but de ce travail n'est pas le développement de ces vérités qui commencent à devenir banales ${ }^{3}$.

Poincaré intervient dans la discussion autour de la notion de l'espace dans le cadre d'un intense débat dans les milieux intellectuels français : la réception des géométries non euclidiennes, la discussion spécifique autour de la question de la géométrie absolue, la réception des travaux de physiologie allemande... Les contributions de Poincaré sur la question de l'espace s'inscrivent dans ce contexte dont l'étude permet d'une part de mieux comprendre la genèse et l'originalité de la réflexion de Poincaré et d'autre part de mieux analyser la réception et le statut du conventionnalisme dans les milieux philosophiques français.

\section{La réception des géométries non euclidiennes en France}

La réception des géométries non euclidiennes en France est pour l'essentiel assurée dans les années 1870 par le mathématicien bordelais Jules Hoüel qui traduit en particulier certains travaux de Bolyai, Lobachevski, Beltrami et une partie de la correspondance entre Gauss et Schumacher. L'objectif principal de la diffusion en France de ces travaux était de convaincre le public mathématicien de la non-contradiction de ces nouvelles géométries. L'intention première de Hoüel n'est certainement pas philosophique ; il publie néanmoins une note (Hoüel 1876) sur le rôle de l'expérience dans les sciences exactes dans laquelle il oppose aux phénomènes réels les phénomènes idéaux qui réfèrent à des êtres de raison. Ces êtres de raison sont dénués de toute existence réelle et sont seulement définis par les propriétés qu'on leur assigne.

Le choix de ces propriétés est a priori arbitraire et soumis à la seule condition de n'offrir rien de contradictoire. Seulement, en vue de

3. Poincaré 1887, p. 215. 
l'utilité que l'on veut tirer de la science abstraite, on s'efforce de les rapprocher autant que possible des propriétés que l'on observe approximativement dans les phénomènes réels ${ }^{4}$.

Hoüel applique alors ce point de vue général à la géométrie. Il admet en préalable l'existence d'un espace immobile dans lequel on sait fixer un point immobile. Une série d'hypothèses qui sont suggérées par l'expérience, comme celle de l'invariabilité des figures, lui permettent de "composer une géométrie complète, exempte de toute contradiction, mais renfermant encore un paramètre indéterminé ${ }^{5}$ :

L'expérience nous conduit à admettre que [ce paramètre] est nul, et comme d'ailleurs cette hypothèse simplifie beaucoup la Géométrie, on a dû adopter exclusivement pour les usages réels la Géométrie enseignée par Euclide, et qui a toujours représenté les phénomènes dans la limite des erreurs d'observation ${ }^{6}$.

La géométrie est donc l'étude théorique d'hypothèses que l'expérience nous "conduit à admettre». Hoüel reconnaît donc la possibilité théorique de référer les phénomènes réels à plusieurs géométries puisque les phénomènes réels ne sont pas susceptibles de déterminations rigoureuses. Néanmoins, la sphère des phénomènes réels a une existence propre et l'expérience nous convainc d'adopter exclusivement la géométrie euclidienne, laquelle a, de plus, l'avantage d'être plus simple. Pour autant, l'étude de la géométrie généralisée n'est pas inutile : elle a une valeur épistémologique puisque d'une part, elle «fixe pour la première fois le rôle que joue dans la science l'hypothèse euclidienne» et que d'autre part, les hypothèses qui s'écartent trop des phénomènes réels peuvent avoir leur utilité pour l'étude d'autres classes de phénomènes. Le débat philosophique portant sur le statut des géométries non euclidiennes rebondit dans le journal fondé par le psychologue Théodule Ribot $^{7}$, la Revue Philosophique, où cette première discussion va en

4. Hoüel 1876, p. XIII.

5. Ce paramètre est la courbure. Toutes les géométries envisagées par Hoüel, Tannery, Calinon et la plupart du temps par Poincaré sont à courbure constante.

6. Hoüel 1876, p. XIV.

7. En tant qu'universitaire, professeur au Collège de France, membre de l'Institut, fondateur et directeur de la Revue philosophique et vulgarisateur tout à la fois, Ribot est le grand initiateur en France de la psychologie expérimentale. Son œuvre s'inscrit dans le programme de fondation d'une nouvelle psychologie, expérimentale, dégagée de toute métaphysique et appuyée sur la physiologie. 
effet en croiser une seconde qui concerne l'importance des sensations et des mouvements dans la formation de la notion d'espace.

Une des contributions essentielle est due à Paul Tannery qui signe deux articles intitulés La géométrie imaginaire et la notion d'espa$c e^{8}$. Comme Hoüel, Tannery introduit la géométrie de Lobachevski comme une généralisation de celle d'Euclide : l'objectif de Lobachevski est présenté comme une tentative de reconstituer la science de l'espace après avoir substitué à l'axiome euclidien des parallèles une hypothèse plus générale; de cette manière, l'axiome de Lobachevski n'est pas envisagé comme une négation, mais comme une généralisation, de l'axiome d'Euclide, et, de ce point de vue, la géométrie euclidienne apparaît comme un cas particulier de celle de Lobachevski. Tannery entame alors une discussion des différentes conceptions philosophiques de l'espace : il résume les réponses que pourraient apporter un philosophe kantien et un philosophe positiviste et empiriste ${ }^{9}$ à la question de la prééminence de la géométrie euclidienne, auxquelles il oppose les vues d'un promoteur des nouvelles géométries. En accord avec ces dernières conceptions, il tente de défendre une position médiane entre les points de vue kantien et positiviste: l'expérience n'est pas suffisante pour nous donner un critère de certitude; en revanche, l'expérience fournit «la matière que l'esprit s'assimile avant de formuler ses axiomes et ses hypothèses " ${ }^{10}$.

Tannery considère "la notion d'espace comme un complexe formé de certains concepts tirés de l'expérience, mais auxquels nous nous attribuons le droit de donner subjectivement des formes différentes $"{ }^{11}$. Ce genre de considérations pourrait presque passer pour une thèse conventionnaliste cependant Tannery ne rejette pas complètement une certaine forme de réalisme: il affirme en effet que parmi tous les complexes logiquement possibles, «il n'y en a jamais qu'un seul qui puisse s'appliquer à l'objet»" ${ }^{12}$. Deux questions se posent alors : d'abord définir un critère absolu pour reconnaître ce complexe unique qui s'applique à l'espace réel, puis déterminer les raisons pour lesquelles «ce complexe objectivable soit tel plutôt que tel autre $»^{13}$. Sur le premier point, Tannery énonce que le seul critère envisageable est l'expérience qui ne pourra jamais

\footnotetext{
8. Cf. Tannery 1876 et Tannery 1877.

9. Tannery identifie le philosophe positiviste au disciple de Comte.

10. Tannery 1876 , p. 450.

11. Ibid.

12. Ibid.

13. Ibid.
} 
donner une certitude absolue, puis conclut en affirmant que même si le point de vue euclidien ne sera probablement jamais contredit par l'expérience, on ne peut pas pour autant en déduire avec les kantiens la nécessité a priori de nos conceptions spatiales. Quant à la seconde question, la réponse reste ouverte aux yeux de Tannery: elle nécessite une étude plus approfondie des nouvelles géométries et une prise en compte des progrès de la physiologie.

Les thèses défendues par Hoüel et Tannery relèvent d'un empirisme modéré ; aucune géométrie n'est a priori privilégiée - en ce sens, la géométrie euclidienne perd son statut de géométrie de référence - néanmoins, la question de la géométrie de l'espace physique reste importante à leurs yeux. Tannery lie ce problème à celui de notre perception; et ainsi certaines caractéristiques du cadre conceptuel dans lequel Poincaré va développer ses propres théories sont-elles déjà définies par Tannery.

Il ne faut pas croire que seuls les tenants de l'empirisme interviennent dans cette discussion. Par exemple, on peut citer Boussinesq (1879 et 1880) qui insiste, au contraire de Tannery, sur le rôle de l'intuition en opposant un sens interne à l'observation externe : certes il reconnaît que cette dernière peut avoir suggéré certaines conceptions géométriques par analogie ou ressemblance imparfaite, mais il soutient qu'elle est « incapable d'atteindre positivement aux conceptions du géomètre entendues dans leur sens rigoureux". Poincaré reprendra ce genre d'arguments pour soutenir que les axiomes de la géométrie ne peuvent être des vérités expérimentales:

Devons-nous donc conclure que les axiomes de la géométrie sont des vérités expérimentales? Mais on n'expérimente pas sur des droites ou des circonférences idéales; on ne peut le faire que sur des objets matériels ${ }^{14}$.

Selon Boussinesq, une des raisons qui montrent la primarité de l'intuition géométrique est l'impossibilité de développer les raisonnements géométriques indépendamment de celle-ci et le fait que les démonstrations géométriques ne se réduisent pas à de simples déductions formelles:

Sans l'intuition, tout raisonnement deviendrait impossible en géométrie, et, probablement, même dans les autres branches des mathématiques ${ }^{15}$.

14. Poincaré 1902, p. 75

15. Boussinesq 1879, p. 363. 
Poincaré utilisera le même argument a contrario pour réfuter que les axiomes de la géométrie soient des «jugements synthétiques a priori, comme disait Kant" :

Ils s'imposeraient alors à nous avec une telle force, que nous ne pourrions concevoir la proposition contraire, ni bâtir sur elle un édifice théorique. Il n'y aurait pas de géométrie non euclidienne ${ }^{16}$.

\section{La géométrie générale de Calinon}

En 1889, Auguste Calinon reprend systématiquement les analyses de Tannery portant sur une géométrie qui engloberait les géométries euclidienne et non euclidienne. En n'usant que de la seule méthode géométrique - c'est-à-dire, pour Calinon, le pur raisonnement - son objectif est de développer, à partir de la définition purement géométrique de la notion de droite générale, une géométrie qu'il appelle "géométrie générale":

La géométrie, ainsi définie [...] n'a plus aucune base expérimentale; elle consiste simplement dans l'application de la méthode dite géométrique à un groupe de formes (lignes ou surfaces) dont la première est soumise à cette condition de permettre l'application de cette méthode.

Comme nous le verrons plus loin, la géométrie ainsi comprise est une science plus générale que la géométrie des anciens ${ }^{17}$.

La définition de la droite euclidienne est la réunion de deux propositions :

a) Une ligne telle que par deux points il n'en passe qu'une.

b) Par un point on ne peut mener à une droite qu'une seule parallèle.

Ainsi le postulat des parallèles n'apparaît-il que "comme la seconde partie d'une définition divisée ${ }^{18}$. Poincaré, parmi d'autres, reprendra cette idée de présenter l'axiome des parallèles comme une définition déguisée de la droite euclidienne.

La géométrie euclidienne est obtenue en appliquant la méthode géométrique à l'ensemble de lignes définies par les propositions $a$

16. Poincaré 1902, p. 74.

17. Calinon 1889, p. 589.

18. Ibid. 
et $b$. Les droites euclidiennes sont, parmi les lignes qui possèdent la propriété $a$, celles qui possèdent en outre la propriété $b$. Il y a donc une notion plus générale de ligne, à savoir celle qui est définie par la seule propriété $a$. Cette notion générale de ligne sert de base à la géométrie générale. Calinon précise qu'il préfère l'appellation géométrie générale à celle plus commune de géométrie non euclidienne parce que sa géométrie n'est pas une négation de la géométrie euclidienne mais comprend au contraire celle-ci comme cas particulier ; aussi bien la géométrie de Lobachevski que celle de Riemann. Calinon décrit donc la géométrie générale comme «l'étude de tous les espaces compatibles avec le raisonnement géométrique $»^{19}$.

La droite générale dépend d'un paramètre et la droite euclidienne correspond à une certaine valeur de celui-ci. Ce paramètre est un paramètre spatial dont chaque valeur est associée à un espace tridimensionnel. Calinon conclut que la géométrie qu'il développe est une "géométrie synthétique d'un nombre infini d'espaces géométriques absolument distincts" et que "la géométrie euclidienne est la géométrie d'un seul de ces espaces ${ }^{20}$. Il précise que la caractéristique de l'espace euclidien (suivant en cela Delbœuf [1860]) est ce qu'il appelle son homogénéité, c'est-à-dire l'existence de similitudes.

La géométrie générale étant obtenue sans le moindre fondement expérimental, la question de la géométrie du monde matériel reste entière. Selon Calinon, l'observation et l'expérience nous amènent à la conclusion que dans les limites de la précision de nos mesures et de nos méthodes expérimentales, "notre espace ne diffère pas de l'espace euclidien ou homogène ${ }^{21}$. Une question peut alors sembler sans réponse : on pourrait en effet considérer comme surprenant que l'expérience concorde avec la notion antérieure (pré-géométrique) que nous possédons de l'espace et de son homogénéité. La réponse de Calinon est très nette: la notion première de l'espace résulte en fait d'une très longue habitude, d'une très longue expérience. L'homogénéité de l'espace n'est ni nécessaire, ni a priori; l'habitude de voir les mêmes faits se reproduire toujours de la même manière nous amène à oublier que ces premières idées sont contingentes. De plus, si l'espace accessible à nos mesures est très petit par rapport à l'univers, la géométrie développée par Riemann

19. Calinon 1891, p. 369.

20. Calinon 1889, p. 593.

21. Ibid., p. 594. 
nous permet de comprendre pourquoi cette partie de l'espace apparaît comme une partie de l'espace euclidien.

À ce point de vue, la géométrie euclidienne n'est [donc] plus qu'une solution très approximative, mais simplifiée, des faits géométriques réels de notre Univers ${ }^{22}$.

Les objectifs théoriques de Calinon sont proches de ceux du conventionnalisme. En effet, en refusant tout caractère a priori ou nécessaire à la géométrie euclidienne, il ne cède pas pour autant face à l'empirisme :

La connaissance que nous avons des formes réelles est expérimentale, par conséquent incomplète et seulement approximative; au contraire les formes idéales de la géométrie résultent de définitions rigoureuses $[\ldots]^{23}$.

Grâce à ces définitions rigoureuses, la connaissance que nous avons des formes géométriques est absolue et complète. Comme Poincaré, Calinon défend une position intermédiaire entre rationalisme kantien et empirisme, mais soutient néanmoins l'idée que l'espace physique possède une géométrie déterminée, laquelle ne peut certes être connue qu'approximativement et peut même évoluer dans le temps. En raison de ce réalisme spatial, Calinon finit donc lui aussi par défendre un empirisme épistémologique dans la mesure où il accorde à l'expérience le rôle primordial dans le choix de la géométrie du monde réel. Comme notre expérience est limitée et que nos constatations expérimentales sont approximatives, il est clair qu' "aucune expérience ne nous autorise à dire que notre espace est rigoureusement euclidien ${ }^{24}$. Tout au plus, Calinon affirme-t-il :

À ce point de vue, la géométrie euclidienne n'est plus qu'une solution très approximative, mais simplifiée, des faits géométriques réels de notre Univers ${ }^{25}$.

La géométrie générale de Calinon donne lieu à une discussion très vive dans les milieux philosophiques français. Les critiques de Renouvier associées à une défense (un peu primaire) du criticisme

22. Calinon 1891, p. 374.

23. Ibid., p. 375.

24. Ibid., p. 373.

25. Ibid., p. 374. 
se concluent par l'affirmation que la négation du postulat d'Euclide est tellement contraire au sens commun que cela prouve sa fausseté. Selon Renouvier, envisager que les lois réelles de notre univers pourraient ne pas être identiques à celles de notre intellect conduit à une sorte de mysticisme. De ce point de vue, le scandale provoqué par les géométries non euclidiennes confirme la futilité des thèses empiristes et le "triomphe de la théorie criticiste de la certitude".

Lechalas trouve au contraire que la géométrie générale de Calinon fournit des arguments contre l'empirisme. Il oppose le réaliste naïf qui pense que l'espace est une réalité extérieure et donc que ses propriétés sont nécessaires et le tenant du criticisme qui "dominant les vaines apparences transporte en lui-même cet espace qui s'imposait tout à l'heure comme une invincible réalité $\|^{26}$. Si pour le criticiste, l'espace perd son "invincible réalité ", il gagne une valeur subjective. Pour Lechalas, l'espace possède une nécessité purement subjective puisque nous ne pouvons pas former d'images appartenant à un espace non euclidien; pour autant, cela ne signifie pas que notre sensibilité n'aurait pas pu être différente. Or du fait du rejet de toute hypothèse expérimentale, la géométrie générale, du point de vue de Lechalas, est donc une "science purement rationnelle, qui ne relève que d'elle-même " ${ }^{27}$. Il n'y a nul besoin de fonder expérimentalement la géométrie; en déniant le caractère contingent de l'espace, la géométrie générale se présente donc davantage comme un argument dirigé contre l'empirisme que contre le criticisme :

Ce qui nous importe, ce n'est point de ne rien devoir aux notions dues à la forme réelle de notre sensibilité, car nous n'avons jamais contesté qu'elles nous sont indispensables pour la constitution d'une géométrie quelconque, mais de reconnaitre que cette forme pourrait être autre qu'elle n'est, tout en conservant des lois rationnelles générales ne relevant que du principe de contradiction. Ces lois constituent la seule base solide sur laquelle puisse s'appuyer le rationalisme dans sa lutte contre l'empirisme, car nous ne croyons pas que les impératifs géométriques soient de nature à inquiéter beaucoup de consciences ${ }^{28}$.

Au contraire de Calinon dont la géométrie générale rejetait tout postulat géométrique et ne reposait selon Lechalas (1890) que sur

26. Lechalas 1890, p. 163.

27. Ibid., p. 162.

28. Ibid., p. 168. 
les axiomes généraux relatifs aux grandeurs et sur des définitions qui ne conduisent pas à des contradictions, Jules Andrade présente sous forme axiomatique une géométrie générale et soutient quant à lui que les axiomes de la géométrie n'ont de signification - c'està-dire de valeur cognitive - que du fait de leur origine expérimentale. Andrade en empiriste conséquent qualifie alors la thèse de Poincaré selon laquelle la question de la vérité de la géométrie n'a pas de sens et qu'en conséquence l'on ne saurait dire que la géométrie euclidienne est vraie et les autres fausses, comme «un point de vue $[\ldots]$ au point philosophique assez particulier ${ }^{29}$.

\section{L'espace, la psychologie et la physiologie}

En 1877, Tannery concluait son second article sur les nouvelles géométries en affirmant que la réponse au problème philosophique de l'espace après l'irruption des géométries non euclidiennes devait être cherchée du côté de la physiologie. Cette conclusion est très révélatrice de l'intensité des débats, en France et ailleurs, autour de la psychologie et de la physiologie, et en particulier des conceptions psychologiques et physiologiques de l'espace, notamment par exemple dans la Revue philosophique, où les travaux anglais et allemands de psychophysiologie sont traduits et abondamment discutés. Il n'est pas question de résumer ici ces discussions mais, outre les livres de Ribot portant sur la psychologie expérimentale anglaise et allemande (Ribot 1870, Ribot 1879a), nous pouvons mentionner les discussions relatives à l'importance psychologique des mouvements, en particulier concernant le "sens musculaire", à la fusion des sensations, et plus généralement au rôle des sens dans la formation de l'idée d'espace (Binet 1880, Delboeuf 1877, 1880, Dunan 1888a, 1888b, Ferré 1885, Gley 1885, Janet 1879, de la Rive 1889). Au-delà de la question de l'innéisme (Hering) et de l'empirisme (Helmholtz), l'ensemble de ces discussions convainc le lecteur que les sens, du moins certains d'entre eux, et les mouvements, de notre corps et des solides qui nous entourent, donnent naissance à certains types d'espace dont les plus importants sont l'espace visuel, l'espace tactile et l'espace moteur. Un autre point important est la capacité de nos nerfs ou de notre esprit de combi-

29. Andrade 1891, p. 432. 
ner les sensations. La composition des sensations de couleurs donne de cette capacité un exemple crucial qui peut être généralisé :

Les lois de compositions trouvées pour les couleurs peuvent donc s'établir en général pour les sensations susceptibles d'être combinées, et elles s'appliquent en particulier aux sensations qui engendrent la notion d'espace ${ }^{30}$.

La perception spatiale est le résultat d'une combinaison de sensations; Delbœuf montre que le phénomène de la vision résulte davantage des propriétés musculaires des yeux que de leurs propriétés optiques. Ainsi, la perception de l'étendue est ajoutée à la sensation visuelle et n'est pas attachée à celle-ci comme une qualité entière et essentielle. Il conclut son raisonnement en affirmant que «l'idée de l'espace est donc propre à tout être doué de motilité» "31:

Il existe un sens des formes de l'étendue, qu'on pourrait appeler le sens géométrique, et qui n'est autre que le sens musculaire. Il est servi par divers instruments plus ou moins parfaits; il emploie [...] des compas plus ou moins précis, à savoir les parties mobiles du corps $^{32}$.

Poincaré reprendra la même thèse au départ de sa théorie de la genèse physio-psychologique de l'espace:

Un être qui ne pourrait pas se mouvoir [...] ne pourrait jamais créer la géométrie, - même si ses sensations étaient variables et même si les objets qui l'entourent étaient mobiles ${ }^{33}$.

Lucien de la Rive (1889), dans un article sur la genèse de la notion d'espace, défend lui aussi l'idée que la genèse de notre notion d'espace relève d'un sens qu'il qualifie de musculaire:

Chaque sens donne lieu à une notion qui lui est spéciale. Il existe donc, dans l'exercice du sens musculaire, une notion qui est pour cette sensation ce qu'est la couleur pour la vue, la résistance pour le toucher, le son pour l'ouie, l'odeur pour l'odorat, la saveur pour le goüt. Ce sens n'est pas qu'apparent, comme pour les autres, parce

30. Tannery 1889 , p. 77.

31. Delbœuf 1877, p. 183. Dans son article "Sur la fusion des sensations semblables ", Delbœuf voit dans la faculté consciente de motilité une condition nécessaire à toute connaissance; cf. Delboeuf 1880, p. 647.

32. Delbœuf 1877, p. 168.

33. Poincaré 1898 , p. 9. 
que la qualité qu'il nous fait connaître du monde sensible, constitue le monde sensible lui-même sous la dénomination d'espace ${ }^{34}$.

Selon Ribot, considérer les mouvements comme une part importante de la vie psychique au même titre que les sensations ou les idées est un des apports majeurs de la psychologie expérimentale. Il défend une psychologie de l'activité motrice ou comme il la dénomme une psychophysiologie. Les mouvements volontaires sont accompagnés de sensations musculaires qui peuvent la plupart du temps être très précisément localisées. En particulier, «c'est le sens musculaire qui nous rend compte à chaque instant de la position de notre corps dans son ensemble, de la position relative des membres, du tronc et de la tête ${ }^{35}$. Dans sa conclusion, Ribot soutient avec les psychologues expérimentalistes que

l'état de conscience qui accompagne certains modes de mouvement musculaire est l'origine de nos perceptions de longueur, hauteur, largeur, forme, position, direction, c'est-à-dire de toutes les déterminations de l'espace ${ }^{36}$.

Le psychologue Eugène Gley (1885) qui défend la même thèse souligne que nous n'avons pas seulement la sensation des mouvements qui sont réellement effectués mais aussi de ceux dont on a seulement l'intention. De la Rive (1889) quant à lui insiste sur une propriété spécifique des sensations de mouvements, à savoir qu'il correspond à chacune de celles-ci la sensation du mouvement opposé. Ces analyses sont reprises par Poincaré lorsqu'il définit les déplacements parmi les changements de sensations comme ceux qui sont susceptibles d'être corrigés par des changements internes, c'est-à-dire des changements volontaires et accompagnés par des sensations musculaires :

Les changements [de sensations] qui sont susceptibles d'être corrigés par un changement interne [...] sont les déplacements ${ }^{37}$.

34. De la Rive 1889 , p. 456.

35. Ribot 1879, p. 377.

36. Ibid., p. 379. Il est clair que Ribot s'inspire en premier lieu fortement des travaux de psychologie allemande, en particulier de l'école d'Helmholtz. Ribot vient de publier son étude sur la psychologie expérimentale allemande (1879b). Il ne faut pas pour autant oublier qu'il avait en 1870 publié une introduction à la psychologie expérimentale anglaise qui elle aussi insistait sur l'importance des sensations musculaires pour la perception spatiale.

37. Poincaré 1898, p. 9. 
Poincaré s'inscrit donc dans une tradition théorique, qui semble dominante en France, selon laquelle la genèse de l'espace relève de l'étude des sensations musculaires associées à nos mouvements; cette théorie est clairement identifiée comme un empirisme:

On sait que l'objet de la théorie communément appelée empiristique est d'établir la genèse de notre idée de l'espace, et l'on sait aussi que tous les philosophes et les physiologistes qui ont adopté cette théorie se sont trouvés d'accord, pour soutenir que l'idée de l'espace ne nous vient, directement au moins, ni de la vue ni du toucher, mais du sens musculaire, ou pour parler avec plus de précision, du sentiment musculaire auquel donnent lieu dans chaque homme les mouvements de son $\operatorname{corps}^{38}$.

Or, comme on va le voir, Poincaré réussit à développer une théorie qui tout en intégrant les travaux les plus modernes de psychologie expérimentale, ne cède rien à l'empirisme philosophique.

\section{La genèse psychophysiologique de l'espace par Poincaré}

Rendant compte de ses vues philosophiques dans l'analyse de ses travaux scientifiques (Poincaré 1901), Poincaré insiste sur les deux moments de sa réflexion sur la théorie de l'espace:

Je me suis demandé quel est le véritable caractère des vérités géométriques et en particulier du postulatum d'Euclide. [...] J'ai recherché également à analyser l'origine psychologique de la notion d'espace ${ }^{39}$.

Dans ces travaux, il maintient une ligne philosophique médiane entre criticisme et empirisme. Il refuse à la fois la doctrine kantienne selon laquelle les axiomes de la géométrie sont synthétiques a priori et les thèses empiristes. Sa réflexion sur la genèse psychophysiologique de l'espace vient appuyer son conventionnalisme et s'inscrit dans ce que l'on pourrait appeler un antiréalisme spatial radical: non seulement, l'espace n'a aucune réalité externe, mais il n'est pas non plus le produit d'une forme de notre sensibilité.

On peut distinguer dans la genèse de la géométrie et de l'espace deux moments : le premier moment est purement psychophysiologique, le second mathématique.

38. Dunan 1888a, p. 136. Charles Dunan en tant que nativiste est opposé aux thèses "empiristiques".

39. Poincaré 1901, p. 127. 
Dans la première partie de sa théorie, Poincaré s'appuie sur les conceptions développées par les psychologues à cette époque : il retient que les «sensations par elles-mêmes n'ont aucun caractère spatial ${ }^{40}$ et que la notion d'espace est obtenue par l'esprit à partir d'une classification des sensations. En 1877, Delbœuf défendait déjà l'idée que «la perception de l'étendue est ajoutée à la sensation mais ne lui est pas attachée comme qualité intégrante et essentielle ${ }^{41}$. De plus, Poincaré suit les conceptions de de la Rive sur la comparaison des sensations. Le point de départ de ce dernier était que «les propriétés de la sensation sont celles d'une quantité sui generis offrant le caractère de la variation continue" (de la Rive 1889, p. 452). Pour de la Rive, la faculté de constater l'existence de cette quantité est implicitement reconnue et constitue la perception.

Poincaré admet un point de départ analogue lorsqu'il affirme l'existence d'une catégorie (qui est une forme de notre entendement) qui préexiste en nous dans laquelle nous classons «les sensations selon leur nature et [rangeons] ensuite celles de même espèce sur une sorte d'échelle suivant leur intensité " ${ }^{42}$. Cette catégorie est appelée par Poincaré espace sensible. Cette catégorie "permet seulement de comparer entre elles des sensations de même espèce et non de les mesurer ${ }^{43}$. La théorie de Poincaré est une tentative de répondre à la question :

Voyons donc comment nous pourrons nous élever de cette catégorie brute $[\ldots]$ à l'espace géométrique ${ }^{44}$.

La réponse à cette question apportée par Poincaré lui permet d'échapper à l'empirisme. En effet, une des thèses essentielles de Poincaré est la différence qu'il fait entre l'espace représentatif ou sensible, entendu comme "le cadre de nos représentations et de nos sensations" (1902, p. 78) et l'espace géométrique. Poincaré

40. Poincaré 1898 , p. 5.

41. Delbœuf 1877, p. 183.

42. Poincaré 1898 , p. 6.

43. Poincaré 1898 , p. 6. Poincaré suit ici les thèses défendues par Helmholtz dans Die Thatsachen in der Wahrnehmung (1879) dont une recension paraît la même année dans la Revue philosophique: "On peut distinguer dans les sensations deux différences essentielles, l'une d'espèce, l'autre de degré. La première sépare, sans permettre de les comparer, les sensations propres aux divers sens. [...] Mais les différences de degré qui existent entre les diverses sensations d'un même sens n'excluent pas entre elles la possibilité d'une comparaison [...]» (Nolen 1879, p. 561-562).

44. Poincaré 1898 , p. 6. 
précise que ces deux types d'espace sont des formes de notre entendement. Si elles ne sont pas indispensables à la formation de nos sensations, ces deux catégories le deviennent pour comparer ou raisonner sur celles-ci (Poincaré 1898, p. 7 et p. 11-12). Il ne faut pas, cependant, les confondre sous prétexte que nous raisonnons sur les corps extérieurs, "comme s'ils étaient situés dans l'espace géométrique $"{ }^{45}$. Poincaré souligne que nos représentations, qui ne peuvent être que des reproductions de sensations, se rangent dans l'espace représentatif.

Nous ne nous représentons pas les corps extérieurs dans l'espace géométrique, mais nous raisonnons sur ces corps, comme s'ils étaient situés dans l'espace géométrique ${ }^{46}$.

Localiser un objet dans l'espace, c'est se représenter les mouvements qu'il faut faire pour atteindre cet objet, autrement dit, se représenter

les sensations musculaires qui les accompagnent et qui n'ont aucun caractère géométrique, qui par conséquent n'impliquent nullement la préexistence de la notion d'espace ${ }^{47}$.

La question reste alors entière de l'origine de l'espace géométrique. Si aucune sensation ne peut par elle-même nous y mener, l'étude des variations des sensations et des «lois suivant lesquelles ces sensations se succèdent" (Poincaré 1902, p. 83) nous conduit à l'idée d'espace géométrique. Poincaré distingue parmi les phénomènes externes (non accompagnés de sensations musculaires) ceux qui sont susceptibles d'être corrigés par un changement interne (accompagné de sensations musculaires). Nous sommes amenés à nous intéresser à cette classe particulière de phénomènes grâce aux mouvements des corps solides matériels. Poincaré appelle ces phénomènes des déplacements et considère que l'étude des lois de ces phénomènes constitue l'objet de la géométrie.

Poincaré commence par classer les déplacements en considérant comme identiques deux déplacements corrigés par un même changement interne, puis montre que "les déplacements forment un groupe".

Ce qui est l'objet de la géométrie, c'est l'étude d'un groupe particulier; mais le concept général de groupe préexiste dans notre esprit

45. Poincaré 1902, p. 82.

46. Ibid., p. 82.

47. Ibid., p. 82 . 
au moins en puissance. Il s'impose à nous, non comme forme de notre sensibilité, mais comme forme de notre entendement ${ }^{48}$.

Seulement, parmi tous les groupes possibles, il faut choisir celui qui sera pour ainsi dire l'étalon auquel nous rapporterons les phénomènes naturels.

L'expérience nous guide dans ce choix qu'elle ne nous impose pas; elle nous fait reconnaître non quelle est la géométrie la plus vraie, mais quelle est la plus commode ${ }^{49}$.

Mais le caractère conventionnel de la géométrie ne s'arrête pas au choix du groupe. Dans On the Fondations of the Geometry (Poincaré 1898) qui est dans un premier temps son article le plus achevé sur la question, Poincaré n'élude pas la question de l'approximation des compensations ou plus généralement celle des expériences par rapport aux lois; l'opération active de l'esprit par laquelle nous imposons à la nature ces compensations ou ces lois aux résultats bruts de l'expérience nécessite en permanence des éléments conventionnels.

Quand l'expérience nous apprend qu'un certain phénomène ne correspond pas du tout aux lois indiquées, nous l'effaçons de la liste des déplacements. Quand elle nous apprend qu'un certain changement ne leur obéit qu'approximativement, nous considérons ce changement, par une convention artificielle, comme la résultante de deux autres changements. Le premier composant est regardé comme un déplacement satisfaisant rigoureusement aux lois dont je viens de parler, tandis que le second composant, qui est petit, est regardé comme une altération qualitative ${ }^{50}$.

Le second moment de la théorie de Poincaré consiste à étudier les propriétés du groupe. En particulier, l'espace géométrique et ses éléments - points, lignes droites, plans... - sont obtenus en considérant et en analysant la structure du groupe et l'action du groupe sur les sous-groupes par les automorphismes intérieurs. Par exemple, la notion de point est obtenue en considérant les sous-groupes de rotations. Dans cette première version de sa théorie, Poincaré n'a nul besoin d'une notion première d'espace, même présentée comme un continuum amorphe et pose que le groupe est premier. Du point de vue de Poincaré, la géométrie précède la notion d'espace.

48. Poincaré 1902a, p. 93-94.

49. Ibid.

50. Poincaré 1898, p. 20. 
On comprend donc pourquoi Poincaré ne retient pas la géométrie générale de Calinon pour sa réflexion philosophique; d'abord, celle-ci présuppose l'existence d'un espace même si celui-ci est amorphe, de plus, avec cette géométrie, il est difficile de développer un point de vue psychophysiologique sur la géométrie ${ }^{51}$.

De ce point de vue, la position de Poincaré est complètement originale par rapport à ses prédécesseurs comme Helmholtz, Lie ou Klein qui tous présupposent l'existence d'un continuum amorphe pour développer leurs conceptions géométriques.

[...] nous devons distinguer dans un groupe la forme et la matière. Pour Helmholtz et Lie la matière du groupe existait avant la forme et en géométrie la matière est une Zahlenmannigfaltigkeit à trois dimensions.

C'est seulement par l'introduction du groupe qu'ils en ont fait une grandeur mesurable, c'est-à-dire un véritable espace. Cependant, l'origine de ce continu non mesurable à trois dimensions reste imparfaitement expliquée $e^{52}$.

Poincaré déduit l'espace et ses propriétés de l'étude du groupe auquel conduit notre expérience des sensations associées aux mouvements internes ou externes. Il montre que même la dimension de l'espace relève de conventions; dans son texte de 1898, Poincaré propose une explication (de caractère mathématique) de la tridimensionalité de l'espace. Il constate que le groupe euclidien sélectionné après de nombreuses conventions peut être vu comme opérant sur un espace de trois, de quatre ou de cinq dimensions et que le choix de la tridimensionalité de l'espace est le résultat d'un choix conventionnel justifié par des considérations de commodité. Malheureusement, son argument est entaché de circularité car le choix du groupe s'appuie sur des considérations fondées sur la classification des groupes de Lie opérant sur les espaces de dimension 3 et admettant un invariant fondamental. En 1903, Poincaré (après ses travaux sur l'Analysis Situs et sa première polémique avec Russell) revient sur cette question dans un article intitulé «l'espace et ses trois dimensions". Il est contraint d'admettre l'existence d'un continuum amorphe de dimension 3 à partir duquel il peut développer sa théorie.

51. Pour les mêmes raisons, Poincaré ne retient pas non plus la géométrie projective ou l'espace projectif. Sur cette question, on peut consulter Nabonnand 2000.

52. Poincaré 1898, p. 29-30. 


\section{L'Analysis situs comme géométrie qualitative}

Dans cet article repris dans la Valeur de la science, Poincaré aborde la question du statut de l'espace de l'Analysis Situs, qu'il présente comme le fond commun des espaces euclidien et non euclidiens. Après avoir insisté une nouvelle fois sur le fait que la géométrie euclidienne ne pouvait constituer une "forme imposée à notre sensibilité" puisque nous pouvons imaginer l'espace non euclidien, il se demande si ce ne serait pas plutôt le continuum amorphe de l'Analysis Situs qui constituerait une forme imposée à notre sensibilité :

Et alors une question se pose: ce continuum amorphe, que notre analyse a laissé subsister, n'est-il pas une forme imposée à notre sensibilité ? Nous aurions élargi la prison dans laquelle cette sensibilité est enfermée, mais ce serait toujours une prison ${ }^{53}$.

Les mêmes questions qui se posaient à propos des vérités de la géométrie euclidienne, se posent de nouveau à propos des théorèmes de l'Analysis Situs. Peuvent-ils être obtenus par un raisonnement déductif? Sont-ce des conventions déguisées? Sont-ce des vérités expérimentales? Sont-ils les caractères d'une forme imposée soit à notre sensibilité, soit à notre entendement ${ }^{54}$ ?

La réponse à ces questions est de nouveau à chercher dans la genèse de la géométrie. Poincaré avait déjà répondu de fait en 1898 à cette question: comme on l'a vu la notion de déplacement est obtenue comme un changement particulier de sensations. Nous faisons comme si les déplacements formaient un groupe continu de transformations mais ce groupe n'opère pas sur l'espace sensible:

Le complexe de nos sensations nous a sans doute pourvus d'une sorte de matière, mais il y a un contraste frappant entre la grossièreté de cette matière et la subtile précision de la forme de notre groupe $^{55}$.

Dans la mesure où la considération de la composition des déplacements nous amène à faire comme si les déplacements constituaient un groupe, nous envisageons la répétition des déplacements et nous sommes conduits à examiner le sous-groupe des multiples d'un déplacement. Lorsque le déplacement $\mathrm{D}$ est très petit, nous

53. Poincaré 1905, p. 59.

54. Ibid., p. 60.

55. Poincaré 1898, p. 30. 
expérimentons ce que Poincaré appelle un continu physique que l'on peut résumer par la formule:

$$
9 \mathrm{D}=10 \mathrm{D}, 10 \mathrm{D}=11 \mathrm{D}, 9 \mathrm{D}<11 \mathrm{D}
$$

Comme Poincaré l'affirme, cette formule répugne à la raison. La question alors se pose de savoir si chaque déplacement appartient à un sous-groupe «discontinu qui nous donnerait l'illusion de la continuité physique parce que nos sens grossiers seraient incapables de discerner deux éléments consécutifs quelconques du faisceau » ou à un sous-groupe dans lequel «les différents déplacements du faisceau seraient commensurables l'un avec l'autre" mais sans qu'il n'y ait rien "qui corresponde aux nombres incommensurables", ou encore à un sous-groupe "parfaitement continu".

Selon Poincaré, la troisième solution s'impose à nous parce que la propriété de continuité s'accorde mieux avec d'autres propriétés du groupe que nous connaissons par expérience. Il précise que c'est la seule raison pour laquelle la continuité s'impose et que «le contraire pourrait être arrivé, [...] que les propriétés du groupe fussent incompatibles avec la continuité». Dans ce cas, nous aurions choisi une autre solution. Le groupe des déplacements sur l'espace sensible et le groupe géométrique obtenu à la fin de la genèse de la géométrie ont donc les mêmes relations que celles qu'entretiennent le continu physique et le continu mathématique :

Nous étudions d'abord la forme [du groupe des déplacements] conformément à la formule du continu physique et comme il y a quelque chose qui répugne à notre raison dans cette formule, nous la rejetons et nous y substituons celle du groupe continu qui en puissance préexiste en nous, mais que nous ne connaissons initialement que par sa forme. La matière grossière qui nous est fournie par nos sensations n'a été qu'une béquille pour notre infirmité et n'a servi qu'à nous forcer à fixer notre attention sur l'idée pure que nous portions en nous ${ }^{56}$.

Le choix de la continuité résulte donc d'un raisonnement conventionnel et l'Analysis Situs étudie donc les propriétés liées à la continuité des espaces mathématiques que l'on obtient à partir des groupes continus.

Cette explication n'est pas entièrement satisfaisante; si elle permet de justifier la continuité du groupe des déplacements et par

56. Poincaré 1898, p. 30. 
là de l'espace, elle n'est pas suffisante pour la suite du programme de Poincaré. En effet, Poincaré utilise la théorie de Lie des groupes continus de transformations et en particulier la classification que ce dernier donne des groupes continus de transformations opérant sur un espace de dimension 3 et admettant un invariant significatif $^{57}$. Il a donc besoin d'introduire une notion de continuum de dimension 3 en usant toujours de conventions.

En 1905, Poincaré propose une autre explication de ce choix en demandant ce que nous "voulons dire quand nous disons qu'un continu mathématique ou un continu physique a deux ou trois dimensions $\|^{58}$. Il introduit alors la notion de coupure pour l'étude des continus physiques. Si deux éléments discernables d'un continu physique peuvent être joints par un chemin d'éléments indiscernables, le continu physique $C$ est dit «d'un seul tenant». Une coupure est un ensemble arbitraire d'éléments de $C$. Selon le choix des coupures, le continu physique restera d'un seul tenant (autrement dit, deux éléments quelconques de $C$ peuvent être joints par un chemin d'éléments indiscernables- continu au sens physique sans quitter $C$ et sans rencontrer la coupure) ou au contraire, il sera divisé par la coupure. Poincaré précise que ces définitions sont suggérées par l'expérience:

On remarquera que toutes ces définitions sont construites en partant uniquement de ce fait très simple, que deux ensembles d'impressions, tantôt peuvent être discernés, tantôt ne peuvent pas l'être ${ }^{59}$.

Un continu physique est dit à une dimension s'il peut être divisé par un certain nombre d'éléments indiscernables. Il est, autrement, dit à plusieurs dimensions ; un continu physique à deux dimensions est un continu qui est divisé par une ou plusieurs coupures à une dimension et ainsi de suite ${ }^{60}$.

Cette définition de la dimension des continus physiques va permettre à Poincaré de rompre le cercle vicieux de sa précédente théorie de la tridimensionalité de l'espace. Dans un premier temps,

57. Voir Engel-Lie 1893, p. 433-434.

58. Poincaré 1905, p. 70.

59. Ibid. p. 73.

60. Poincaré précise que selon lui cette définition est identique à celle des géomètres qui définissent les surfaces comme les limites des volumes et les lignes comme les limites des surfaces. Il ajoute qu'il vient de donner à la notion de dimension "une forme applicable non au continu mathématique, mais au continu physique, qui est seul susceptible de représentation» (1905, p. 64). 
il abandonne une partie de cette théorie. Puisque le continu (physique) des déplacements a six dimensions et que ce résultat est acquis par l'expérience ${ }^{61}$, on pourrait reprendre le précédent raisonnement et montrer que l'on peut déduire la tridimensionalité de l'espace, mais une telle théorie ne saurait être satisfaisante car elle ne respecte pas la hiérarchie de nos perceptions géométriques:

[...] et quand nous aurons montré comment la notion de ce continu peut se former et comment on peut en déduire celle de l'espace, on pourrait toujours se demander pourquoi l'espace à trois dimensions nous est beaucoup plus familier que ce continu à six dimensions, et douter par conséquent que ce soit par ce détour, que s'est formée dans l'esprit humain la notion d'espace ${ }^{62}$.

Poincaré propose une nouvelle théorie qui explique que nous sommes guidés par l'expérience vers l'étude des groupes de transformations des espaces à trois dimensions. L'espace essentiel sera celui associé au sens du toucher, l'espace tactile, dont il montre qu'une des conditions nécessaires à son établissement est que les impressions tactiles ne soient pas altérées par les sensations musculaires associées à nos mouvements. Chacun des espaces associés à nos doigts est un continu physique à trois dimensions dont Poincaré montre que l'on est, de nouveau sur la base d'expériences, amené à les considérer comme semblables entre eux ainsi qu'à l'espace visuel.

En montrant le rôle essentiel de l'expérience dans la genèse de la géométrie, Poincaré n'a pas pour autant sombré dans un empirisme béat et stérile. En effet, comme il le précise lui-même :

l'expérience ne prouve pas que l'espace a trois dimensions; elle nous prouve qu'il est commode de lui en attribuer trois, parce que c'est ainsi que le nombre des coups de pouce est réduit au minimum ${ }^{63}$.

61. En 1898, Poincaré utilisait le fait que le groupe des déplacements était à six paramètres. Il justifiait cette affirmation en disant que l'expérience nous permettait de nous rendre compte que tout déplacement infinitésimal pouvait être décomposé le long de six déplacements particuliers (théorie de Lie). En 1905, il assure qu'«il serait aisé de décrire les expériences par lesquelles nous pourrions arriver à ce résultat. On verrait qu'on peut dans ce continu pratiquer des coupures qui le divisent et qui sont des continus ; qu'on peut diviser ces coupures elles-mêmes par d'autres coupures du second ordre qui sont encore des continus, et qu'on ne serait arrêté qu'après les coupures du sixième ordre qui ne seraient plus des continus " (1905, p. 102).

62. Poincaré 1905, p. 102.

63. Ibid. p. 120. 
Le choix des conventions est justifié selon les cas par des considérations d'accommodement à notre expérience, soit par des raisons de commodité pratique ou mathématique. Le choix d'une convention ouvre un espace de possibilité d'actions, parmi lesquelles celles de donner des significations, de définir de nouveaux objets. Mais, comme Poincaré ne cesse de le rappeler, si elle n'est pas arbitraire, une convention n'est pas, pour autant, imposée par l'expérience. Ces thèses sont originales par rapport aux travaux concernant l'espace tant en philosophie qu'en physiologie; pour autant elles s'inscrivent dans un mouvement qui revenant sur un siècle de criticisme et de réalisme spatial, met les mouvements de notre corps et des solides aux fondements de la réflexion sur l'espace.

Philippe NABONNAND

Nancy-Université LPHS-Archives Poincaré, UMR 7117

\section{Références bibliographiques}

ANDRADE J. (1890), "Les bases expérimentales de la géométrie», La Revue philosophique, 30, p. 406-411.

ANDRADE J. (1891), "Les bases expérimentales de la géométrie euclidienne ", La Revue philosophique, 31, p. 430-432.

BineT A. (1880), "De la fusion des sensations semblables", La Revue philosophique, 9, p. 284-294.

BOUSSINESQ J. (1879), "Sur le rôle et la légitimité de l'intuition géométrique ", La Revue philosophique, 8, p. 357-370.

BoussinesQ J. (1880), "Sur l'impossibilité d'arriver aux notions géométriques par une simple condensation d'un grand nombre de résultats de l'expérience", La Revue philosophique, 9, p. 444-449.

CALINON A. (1889), "Les espaces géométriques (1)", La Revue philosophique, 28, p. 588-595.

CALINON A. (1891), "Les espaces géométriques (2)", La Revue philosophique, 32, p. 368-375.

Delbouf J. (1860), Prolégomènes philosophiques de la géométrie et solution des postulats, Liège, J. Desoer.

DelbéfF J. (1877), "Du rôle des sens dans la formation de l'idée d'espace», La Revue philosophique, 4, p. 167-184.

Delbeuf J. (1880), "Sur la fusion des sensations semblables ", La Revue philosophique, 10, p. 644-648. 
DUNAN Ch. (1888a), "L'espace visuel et l'espace tactile», La Revue philosophique, 25, p. 134-169.

DUNAN Ch. (1888b), "L'espace visuel et l'espace tactile», La Revue philosophique, 25, p. 591-619.

FERRÉ Ch. (1885), "Sensation et mouvement», La Revue philosophique, 20, p. 337-368.

GLEY Eu. (1885), "Le "sens musculaire" et les sensations musculaires», La Revue philosophique, 20, p. 601-610.

Helmholtz H. von (1856), Handbuch der physiologischen Optik, $2^{\mathrm{e}}$ éd., Leipzig (Hambourg, 1886-1889); E. Javal et N.Th. Klein (trad.), Optique physiologique, Paris, Masson, 1867.

Helmholtz H. von (1879), Die Thatsachen in der Wahrnehmung, Berlin. HoüEL J. (1876), "Sur le rôle de l'expérience dans les sciences exactes", Mémoire de la Société des sciences physiques et naturelles de Bordeaux, $2^{\mathrm{e}}$ série, $1, \mathrm{XIII}-\mathrm{XV}$.

JANET P. (1879), "De la perception visuelle de la distance», La Revue philosophique, 7, p. 1-17.

LECHALAS G. (1889), "La géométrie générale », La Critique philosophique, 5, p. 217- 231 .

LECHALAS G. (1890), "La géométrie générale et les jugements synthétiques a priori ", La Revue philosophique, 30, 157-169.

NABONNAND Ph. (2000a), "Les recherches sur l'œuvre de Poincaré : État des lieux (1990-2000)", La Gazette des mathématiciens, 85, p. 33-54.

Nabonnand Ph. (2000b), "La polémique entre Poincaré et Russell. Au sujet du statut des axiomes de la géométrie", Revue d'histoire des mathématiques, 6, p. 219-269.

NABONNAND Ph. (2005), "Bibliographie des travaux sur l'œuvre de Poincaré (2001-2005)", Philosophia Scientiae, 9, p. 195-206.

NOLEN D. (1879), "Analyse et comptes rendus - Helmholtz, Les faits dans la perception", La Revue philosophique, 7, p. 561-567.

POINCARÉ H. (1887), "Sur les hypothèses fondamentales de la géométrie ", Bulletin de la Société mathématique de France, 15, p. 203-216; OC XI, Paris, J. Gabay, 2005, p. 75-91.

PoINCARÉ H. (1898), "On the Foundations of Geometry», The Monist, IX, 1-43 (trad. Louis Rougier, Des Fondements de la géométrie, Paris, Chiron, 1921 ; cité d'après la rééd. dans L'opportunisme scientifique, L. Rollet (éd.), Bâle, Birkhäuser, 2002, p. 5-31).

POINCARÉ H. (1901), "Analyse des travaux scientifiques de Henri Poincaré faite par lui-même", rééd. in Acta Mathematica, 38, 1921, p. 36135.

PoInCARÉ H. (1902), La Science et l'Hypothèse, Paris, Flammarion; cité selon la rééd., Paris, Flammarion, 1968.

POINCARÉ H. (1903a), "L'espace et ses trois dimensions", Revue de métaphysique et de morale, 11, p. 281-301. 
POINCARÉ H. (1903b), "L'espace et ses trois dimensions ", Revue de métaphysique et de morale, 11, p. 407-429.

PoINCARÉ H. (1905), La Valeur de la science, Paris, Flammarion; cité selon la rééd., Paris, Flammarion, 1970 (en particulier chap. III et IV).

RENOUVIER Ch. (1889), "La philosophie de la règle et du compas", $L a$ Critique philosophique, 5, p. 337-348.

Riвот T. (1870), La Psychologie anglaise contemporaine (école expérimentale), Paris, Ladrange.

Riвот T. (1878), "Les théories allemandes sur l'espace tactile», La Revue philosophique, 6, p. 130-145.

Ribot T. (1879a), La Psychologie allemande contemporaine (école expérimentale), Paris, Alcan.

RiвоT T. (1879b), "Les mouvements et leur importance psychologique», La Revue philosophique, 8, p. 371-386.

RIVE L. de la (1889), "Sur la genèse de la notion d'espace", La Revue philosophique, 27, p. 432-462.

TANNERY P. (1876), "La géométrie imaginaire et la notion d'espace (1)", La Revue philosophique, 2, p. 433-451.

TANNERY P. (1877), "La géométrie imaginaire et la notion d'espace (2)", La Revue philosophique, 3, p. 553-575.

TANNERY P. (1889), "Revue générale - Philosophie mathématique et psychophysique ", La Revue philosophique, 27, p. 73-82. 